\section{Cognitive behaviour therapy for the chronic fatigue syndrome}

\section{Evening primrose oil and magnesium have been shown to be effective}

EDITOR,-In their paper on cognitive behaviour therapy for the chronic fatigue syndrome Michael Sharpe and colleagues state that many pharmacological treatments have been suggested but none are of proved value. ${ }^{1}$ Last year Lewith stated that the only two treatments that had been properly evaluated were evening primrose oil and magnesium by injection. ${ }^{2}$ Intramuscular magnesium supplements have been given to patient with low red cell magnesium in a double blind placebo controlled trial; myalgia and fatigue improved in about $70 \%$ of subjects. ${ }^{3}$ Evening primrose oil has been used to treat myalgic encephalomyelitis and is the only other treatment that has been adequately tested in a controlled trial. High doses in randomised controlled trials have been shown to have a significant effect in $70-80 \%$ of patients with myalgic encephalomyelitis or the chronic fatigue syndrome. ${ }^{4} \mathrm{I}$ would be interested to hear Sharpe and colleagues' comments about these papers.

S A CHILTON

Martonside Medical Centre

Middlesbrough,

Cleveland TS4 3BU

1 Sharpe M, Hawton K, Simkin S, Surawy C, Hackmann A, Klimes I, et al. Cognitive behaviour therapy for the chronic fatigue syndrome: a randomised controlled trial. $B M 9$ 1996;312:22-6. (6 January.)

Lewith G. Chronic fatigue syndrome. Update 1995;50:765.

3 Cox IM, Campbell MJ, Dowson DI. Red blood cell magnesium levels and the chronic fatigue syndrome $(\mathrm{ME})$, a case control study and a randomised controlled trial. Lancet 1990;337:757-60.

4 Behan PO, Behan WMH, Horrobin D. The effect of high doses of essential fatty acids in the post-viral fatigue syndrome. Acta Neurol Scand 1990;82:209-16.

\section{Good general care may offer as much} benefit as cognitive behaviour therapy

EDITOR,-Successful outcomes have been reported from controlled clinical trials of an eclectic range of treatments-from immunotherapy to magnesium supplementation-for the chronic fatigue syndrome. ${ }^{1}$ Unpublished data suggest that equal success can be achieved with some forms of alternative therapy (for example, homoeopathy) when patients believe strongly in the approach. Most physicians, however, continue to view all such results with healthy scepticism. An equally cautious view needs to be taken when assessing Michael Sharpe and colleagues' study of cognitive behaviour therapy. ${ }^{2}$ In a disorder that is almost certainly heterogeneous in nature, two important questions need to be answered before we can conclude that cognitive behaviour therapy is of value.

Firstly, does cognitive behaviour therapy offer any more benefit than good general care, support, and use of drugs for symptomatic relief (particularly for alleviating insomnia, myalgia and any coexistent depression), which is increasingly being provided by general practitioners and the primary health care team? The protocol used in a controlled study of cognitive behaviour therapy in Australia may help with the answer to this. ${ }^{3}$ Control patients in Sharpe and colleagues' study, who failed to show any significant improvement after one year, received "no further specific explanation or advice" apart from being advised to increase their level of activity gradually. By comparison, the control group in the Australian study-whose improvement was similar to that of those receiving cognitive behaviour therapy-were given medical care by a unit with recognised skill in all aspects of managing the chronic fatigue syndrome.

Secondly, are patients who benefit from cognitive behaviour therapy a subgroup with appreciable coexistent psychiatric morbidity? Although Sharpe and colleagues' study contained a high proportion $(67 \%)$ of patients with depression, anxiety, or somatisation disorder, no useful information is provided on whether these factors may have influenced outcome. Findings from an American controlled study of cognitive behaviour therapy suggest, however, that a subset of patients with the chronic fatigue syndrome, who have high levels of depressive symptoms and maladaptive thinking regarding their fatigue, are the ones who can really benefit from this type of behavioural intervention.

The apparent success rates for pharmacological, psychological, and alternative treatments in the chronic fatigue syndrome indicate that assessment of each patient remains the most important determinant when approaches to management are being considered. Until more is known about the precise pathoaetiology of the syndrome this is likely to remain the case.

CHARLES SHEPHERD

ME Association,

Honorary medical advise

Stanford le Hope,

Essex SS17 OHA

1 Shepherd C. Myalgic encephalomyelitis: post-viral fatigue syndrome. Guidelines for the care of patients. 2nd ed Stanford le Hope: ME Association, 1995.

2 Sharpe M, Hawton K, Simkin S, Surawy C, Hackmann A Klimes I, et al. Cognitive behaviour therapy for the chronic fatigue syndrome: a randomised controlled trial. $B M$ 1996;312:22-6. (6 January.)

3 Lloyd AR, Hickie I, Brockman A, Hickie C, Wilson A, Dwyer J, et al. Immunologic and psychologic therapy for patients with chronic fatigue syndrome: a double-blind, placebocontrolled trial. Am $\mathcal{F}$ Med 1993;94:197-203.

4 Friedberg F, Krupp LB. A comparison of cognitive behaviou trearment for chronic fatigue syndrome and primary depression. Clin Infect Dis 1994;18 (suppl):105-9.

\section{Patients were not representative of all}

\section{patients with the syndrome}

EDITOR,-Michael Sharpe and colleagues conclude that cognitive behaviour therapy leads to a sustained reduction in functional impairment for patients with the chronic fatigue syndrome. ${ }^{1}$ The levels of disability of the 60 patients who took part in the study suggest, however, that these patients do not represent a comprehensive cross section of patients with the syndrome. The 60 patients scored $60-78$ on the Karnofsky scale assessing disability and so represent a different population from the 143 patients reported on by Case History Research on ME (myalgic encephalomyelitis), who would have scored 30-60 (R Gibbons et al, first world congress on chronic fatigue syndrome and related disorders, Brussels, Nov 1995). Fifty nine of these 143 patients reported functional deterioration after sustained, incrementally increased physical exertion.

The authors did not assess other symptoms common in the chronic fatigue syndrome, such as pain, nausea, muscle weakness, or balance problems - a measure of the reduction of which was taken as a standard for "success" in an earlier trial. ${ }^{2}$ The lack of evidence of significant changes in other measures besides "the principal complaint of severe fatigue" in the authors' study tends to diminish the validity of their conclusions.

If functional capacity alone is to be assessed in a trial a validated instrument should be used. The Karnofsky scale is not sensitive enough to measure function since a value of 80 implies "normal activity with effort; some signs or symptoms of disease." But what does the phrase "normal activity" mean? Does it mean being able to get up, dress, and walk at a level sufficient to maintain (unemployed) independence or does it mean an ability to perform all these activities together with a return to full time employment and participation in sport?

Given the heterogenous nature of the chronic fatigue syndrome, we are concerned at the apparent enthusiasm for cognitive behaviour therapy, which may be helpful for patients who have factors such as depression or maladaptive behaviour (too much bed rest) but may in the long term be detrimental to a specific subgroup of patients. We note that in Sharpe and colleagues' trial the condition of four of the 30 patients given cognitive behaviour therapy had deteriorated after 12 months. Could these patients have a distinct type of the chronic fatigue syndrome, meeting the original clinical criteria for myalgic encephalomyelitis, ${ }^{3}$ and be at risk from cognitive behaviour therapy?

RAY GIBBONS Researche ANNE MACINTYRE Researche CHRIS RICHARDS Researcher

Case History Research on ME (CHROME)

3 Britannia Road

London SW6 $2 \mathrm{HI}$

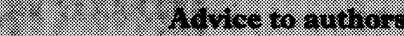

We receive more letters than we can publish: we can currently accept only about one third. We prefer short letters that relate to articles published within the past four weeks. Letters received after this deadline stand less chance of acceptance. We also publish some "out of the blue" letters, which usually relate to matters of public policy.

When deciding which letters to publish we favour originality, assertions supported by data or by citation, and a clear prose style. Wit, passion, and personal experience also have their place.

Letters should have fewer than 400 words and no more than five references (including one to the BMJ article to which they relate); references should be in the Vancouver style. We welcome pictures.

Letters should be typed and signed by each author, and each author's current appointment and address should be stated. We encourage you to declare any conflict of interest.

Please enclose a stamped addressed envelope if you would like to know whether your letter has been accepted or rejected.

Letters will be edited and may be shortened. 
Sharpe M, Hawton $K$, Simkin S, Surawy C, Hackmann A Klimes I, et al. Cognitive behaviour therapy for the chronic fatigue syndrome: a randomised controlled trial. $B M$ 1996;312:22-6. (6 January.)

2 Lloyd AR, Hickie I, Brockman A, Hickie C, Wilson A, Dwyer J, et al. Immunologic and psychologic therapy for patients with chronic fatigue syndrome: a double-blind, placebocontrolled trial. Am f Med 1993;94:197-203.

3 Ramsay AM. Myalgic encephalomyelitis and postviral fatigue states: the saga of Royal Free disease. 2nd ed. London: Gowe Medical, 1989:29-31.

\section{Use an interdisciplinary approach}

EDITOR,-From their randomised trial in the chronic fatigue syndrome Michael Sharpe and colleagues conclude that cognitive behaviour therapy is more effective than "medical care" in improving day to day function. ${ }^{1}$ It is not clear that the data presented justify this conclusion.

Firstly, the authors do not compare like with like: the group given cognitive behaviour therapy received 16 hours of therapy while the "medical" group received no intervention. Secondly, the "medical" group of patients were "advised to increase their level of activity by as much as they felt able," which may have had adverse effects if the activity was unsupervised and inappropriate. This could have affected the results by making the group given cognitive behaviour therapy seem to improve by more than they did. Thirdly, all patients, and particularly those with the chronic fatigue syndrome, need detailed discussion of their problems. Many doctors will not have been aware that in providing such discussion-surely the duty of all doctors - they were in part providing cognitive behaviour therapy.

Perhaps it is inappropriate to complain that the authors did not address the physical problems of the chronic fatigue syndrome as this was not part of their remit. However, physical factors, including perfusion changes ${ }^{3}$ and erythrocyte rigidity, are present. ${ }^{4}$ Over 100 papers in the literature show that stress damages the immune system ${ }^{5}$ : cognitive behaviour therapy is likely to be helpful.

Surely the best way of treating this complex disorder is by using an interdisciplinary approach rather than the limited potential of any single modality. This study might have been more productive if the medical group had undergone a comparable and appropriate programme of physical management.

K K EATON

Princess Margaret Hospital,

Consultant

Windsor

Berkshire SL4 3S

1 Sharpe M, Hawton K, Simkin S, Surawy C, Hackmann A Klimes I, et al. Cognitive behaviour therapy for the chronic fatigue syndrome: a randomised controlled trial. $B M$ 1996;312:22-6. (6 January.)

2 Lane RJM, Burgess RP, Flint J, Riccio M, Archard LC. Exercise responses and psychiatric disorder in chronic fatigue syndrome. BMF 1995;311:544-5.

3 Costa DC, Brostoff J, Douli V, Ell PJ. Brain stem hypoperfusion in patients with myalgic encephalomyelitis hypoperfusion in patients with myalgic

4 Simpson LO. The role of non discocyte erythrocytes in the pathogenesis of myalgic encephalomyelitis/chronic fatigue pathogenesis of myalgic encephalomyelitis/chronic fatigue syndrome. In: Hyde B, Godstein J, Levin P, eds. The clinical
and scientific basis of myalgic encephalomyelitis/chronic fatigue and scientific basis of myalgic encephalomyelitis/chronic fatigue
syndrome. Ottawa: Nightingale Research Association syndrome. Ot

5 Irwin M, Daniels M, Bloom ET, Smith TL, Weiner H. Life events, depressive symptoms and immune function. $A m$ Psychiatry 1987;144:437-41.

\section{Essential elements of the treatment must be identified}

EDrToR,-We have several practical and theoretical concerns about Michael Sharpe and colleagues' study of cognitive behaviour therapy in the chronic fatigue syndrome. ${ }^{1}$ The authors managed to obtain almost $100 \%$ uptake of treatment and compliance among patients who were attending an infectious diseases clinic and were strongly convinced that their chronic fatigue had a physical cause. We would struggle to engage our patients similarly, even with two hours for an initial appointment, and we could not offer them anything approaching an hour of treatment week for four months. The difference between what was provided in the study and what clinicians can routinely offer their patients makes it important to identify the essential elements of the treatment.

The package given included cognitive techniques such as "question[ing] a simple disease explanation," "strategies to reduce excessive perfectionism and self criticism," and a problem solving approach of "gradual and consistent increases in activity." The continuing improvement after the end of treatment is unusual for the cognitive psychotherapies and suggests that the behavioural component was most effective. We find it puzzling, therefore, that the authors attribute the beneficial effects of treatment to " specific effect on illness perpetuating beliefs and coping behaviour," particularly as these attitudes did not change substantially. The patients would inevitably report less avoidance of exercise if they were complying with the study. After treatment at least half of the patients still believed that the illness was physical (from tables 2 and 5), and the vast majority still applied the damaging label of "myalgic encephalomyelitis" 2 to their condition.

Although the authors rightly state that their results tell us little about aetiology, they will inevitably be taken (at least by some people) to mean that the condition is primarily psychological-particularly as the authors have stated elsewhere that the chronic fatigue syndrome "does not reflect a particular biological process, but rather specific psychologic and behavioural mechanisms." ${ }^{3}$ Notwithstanding an artificial distinction between biology and behaviour, such "psychologisation" of the illness ignores increasing evidence of specific neurobiological abnormalities. ${ }^{45}$

If, as we suspect, the behavioural component of the treatment proves to be the essential therapeutic element then this will provide more specific information for hypotheses on the causes of the chronic fatigue syndrome and allow the most efficient use of an effective but time consuming treatment.

STEPHEN M LAWRIE

Department of Psychiatry,

University of Edinburgh,

Royal Edinburgh Hospital,

Edinburgh EH10 5H

Hairmyres Hospital,

Glasgow G75 8RG

1 Sharpe M, Hawton K, Simkin S, Surawy C, Hackmann A Klimes I, et al. Cognitive behaviour therapy for the chronic fatigue syndrome: a randomised controlled trial. $B M$ 1996;312:22-6. (6 January.)

2 Lawrie SM, Pelosi AJ. Chronic fatigue syndrome: prevalence and outcome. $B M F$ 1994;308:732-3.

3 Surawy C, Hackmann A, Hawton K, Sharpe M. Chronic fatigue syndrome: a cognitive approach. Behav Res Ther 1995;33:535-44.

4 Demitrack MA, Dale JK, Straus SE, Laue L, Listwak SJ, Kruesi MJP, et al. Evidence for impaired activation of the hypothalamic-pituitary-adrenal axis in patients with chronic fatigue syndrome. I Clin Endocrinol Metab 1991;73:1224 34.

5 Bearn JA, Allain T, Coskeran P, Munro N, Butler J, McGrego $A$, et al. Neuroendocrine responses to $\mathrm{d}$-fenfluramine and insulin-induced hypoglycaemia in chronic fatigue syndrome. Biol Psychiatry 1995;37:245-52.

\section{Cognitive behaviour therapy should be compared with placebo treatments}

EdrToR,-Lest Michael Sharpe and colleagues' paper lends respectability to the notion that the chronic fatigue syndrome is a diagnostic entity or suggests that cognitive behaviour therapy has any value specific to the condition, ${ }^{1}$ I wish to make three points.

Firstly, the disorder that the authors treated is heterogeneous, the only defining criteria used being fatigue, impaired daily activities, and the absence of signs of physical disease or "severe depression." Claims for a specific effect in any diffuse symptom complex are dangerous. Quinine is effective in many cases of cramp, but neither the symptom nor the benefit is specific.

Secondly, cognitive behaviour therapy and any comparable substitute were denied the control patients, who were therefore matched only on pretreatment criteria regarding their clinical state and not controlled in respect of a comparable treatment. Despite the authors' claim for a "specificity of treatment effect" the benefits shown are consistent with the provision of much attention, encouragement, and a positive attitude to the nature of the illness and the strategies to counter it.

Thirdly, Sharpe and colleagues did not compare cognitive therapy with other treatments or placebo, and the improvements (including persistence after the treatment had ended) are compatible with a placebo effect. ${ }^{3}$ The "return to normal functioning (albeit with continuing fatigue) in most cases" is typical of the outcome for many such patients managed by other means. Before this expensive and time consuming remedy is generally applied, a formal comparison of cognitive therapy with placebo treatments is needed.

JIM PEARCE

Anlaby,

Consultant neurologis

Hull HU10 7BG

1 Sharpe M, Hawton K, Simkin S, Surawy C, Hackmann A Klimes I, et al. Cognitive behaviour therapy for the chronic fatigue syndrome: a randomised controlled trial. $B M J$ fatigue syndrome: a randomis

2 Woods TO, Goldberg DP. Psychiatric perspectives: an overview. Br Med Bull 1991;47:908-18.

3 Pearce JMS. The placebo enigma. Qf Med 1995;88:215-20.

\section{Patients' beliefs about their illness were} probably not a major factor

EDITOR,-Michael Sharpe and colleagues' study confirms that the best medical advice for patients with the chronic fatigue syndrome is not "nothing can be done" or that "the disease will burn itself out." The study produced improvement in $73 \%$ of the patients, which is comparable to the $80 \%$ improvement produced by my management techniques. ${ }^{2} 3$ Interestingly, my approach seems to be fundamentally different from that of Sharpe and colleagues.

Two previous studies of cognitive behaviour therapy in patients with the chronic fatigue syndrome showed no benefit over ordinary medical management. ${ }^{45}$ What is meant by cognitive behaviour therapy may go some way to explaining this apparent discrepancy between previous studies and that of Sharpe and colleagues. Most of us would agree that "inaccurate and unhelpful beliefs, ineffective coping behaviour, negative mood states, social problems, and pathophysiological processes all interact to perpetuate the illness." I I would also agree with Sharpe and colleagues' next statement that "treatment aims at helping patients to re-evaluate their understanding of the illness and to adopt more effective coping behaviours." Sharpe and colleagues conclude that patients gain benefit from cognitive behaviour therapy because it reduces their beliefs that illness is mainly physical, the cause is a virus, the illness is myalgic encephalomyelitis, and exercise should be avoided. $M y$ approach in the initial management aims to increase the patients' beliefs in these areas. ${ }^{3}$ So how could our results be similar? 